\title{
Correction to: Understanding the Stochastic Partial Differential Equation Approach to Smoothing
}

\author{
David L. MilleR@, Richard GLENNIE@, and Andrew E. SEATON®
}

Correction to: JABES

https://doi.org/10.1007/s13253-019-00377-z

Unfortunately, in the original publication of the article, several definitional mistakes crept into the manuscript during writing. A complete, corrected version of the manuscript can be found at https://arxiv.org/abs/2001.07623. The results of the paper are unchanged, but the errors and their corrections are listed below.

1. Definition of $c(x, y)$ in Sect. 2.3. The Matérn formulation should be

$$
c(x, y)=\frac{2^{1-v}}{(4 \pi)^{d / 2} \kappa^{2 v} \tau^{2} \Gamma(\nu+d / 2)}(\kappa\|x-y\|)^{v} K_{\nu}(\kappa\|x-y\|)
$$

2. At end of second paragraph in Sect. 2.3 should be $\alpha=v+d / 2$.

3. In Sect. 3.1, the thin plate penalties should have squared integrands, i.e., $J(\boldsymbol{\beta}, \lambda)=$ $\lambda \int\left(\partial^{2} f / \partial x^{2}\right)^{2}+2\left(\partial^{2} f / \partial x \partial y\right)^{2}+\left(\partial^{2} f / \partial y^{2}\right)^{2} \mathrm{~d} x \mathrm{~d} y$.

4. In Sect. 3.3, in paragraph 2, the expression should be $\langle D f, D f\rangle=\tau^{2}\left(\kappa^{4}\langle f, f\rangle+\right.$ $\left.2 \kappa^{2}\langle\nabla f, \nabla f\rangle+\langle\Delta f, \Delta f\rangle\right)$. Following on from that, the definitions of $\boldsymbol{G}_{1}, \boldsymbol{G}_{2}$ should be $\left\langle\nabla \psi_{i}, \nabla \psi_{j}\right\rangle$ and $\left\langle\Delta \psi_{i}, \Delta \psi_{j}\right\rangle$, respectively.

Publisher's Note Springer Nature remains neutral with regard to jurisdictional claims in published maps and institutional affiliations.

The original article can be found online at https://doi.org/10.1007/s13253-019-00377-z.

D. L. Miller $(\varangle) \cdot$ R. Glennie - A. E. Seaton, Centre for Research into Ecological and Environmental Modelling and School of Mathematics and Statistics, University of St Andrews, St Andrews, Fife, Scotland, UK

(E-mail:dave@ninepointeightone.net).

(C) 2020 The Author(s)

Journal of Agricultural, Biological, and Environmental Statistics, Volume 25, Number 2, Pages 276

https://doi.org/10.1007/s13253-020-00383-6 